\title{
Severe adenomyosis with unexpectedly high CA-125: report of a rare case
}

\author{
Ibrahim A. Abdelazim ${ }^{1,2}$, Mohannad AbuFaza², Mohamed E.S. Hamed ${ }^{1}$, Yerbol Bekmukhambetov³, \\ Gulmira Zhurabekova ${ }^{4}$, Svetlana Shikanova ${ }^{5}$ \\ ${ }^{1}$ Department of Obstetrics and Gynecology, Ain Shams University, Cairo, Egypt \\ 2Department of Obstetrics and Gynecology, Ahmadi Kuwait Oil (KOC) Company Hospital, Ahmadi, Kuwait \\ ${ }^{3}$ Department of Oncology, West Kazakhstan Marat Ospanov Medical University, Aktobe, Kazakhstan \\ ${ }^{4}$ Department of Normal and Topographic Anatomy, West Kazakhstan Marat Ospanov Medical University, Aktobe, Kazakhstan \\ ${ }^{5}$ Department of Obstetrics and Gynecology No. 1, West Kazakhstan Marat Ospanov Medical University, Aktobe, Kazakhstan
}

\section{Abstract}

A 46-year-old woman presented to the emergency department with abdominal pain and abnormal uterine bleeding over the last year. She had a history of failed cyclic progesterone with tranexamic acid over the last 3 months to control her bleeding attacks. She had a tender pelvi-abdominal mass of 28 weeks' gestation size. Therapeutic curettage was performed, followed by correction of her iron deficiency anemia with intravenous iron.

The trans-abdominal ultrasound of the pelvi-abdominal mass showed asymmetrical myometrial thickening, predominantly its anterior wall, with myometrial hyperechoic regions surrounded by hypoechoic areas suggestive of adenomyosis uterus for magnetic resonance imaging (MRI) evaluation.

The MRI evaluation confirmed the diagnosis of adenomyosis uterus and showed diffuse asymmetrical uterine adenomyosis, predominantly its anterior myometrial wall, with an ill-defined endo-myometrial junction. Total abdominal hysterectomy with bilateral salpingectomy and bilateral ovarian conservation was performed after departmental approval and the patient's consent.

The routine pre-operative investigations were completely normal except the cancer antigen (CA-125) which was unexpectedly high (1658 and $1046 \mathrm{IU} / \mathrm{ml}$ repeated twice two days apart). The histological examination of the surgical removed uterus confirmed the diagnosis of adenomyosis uterus and the pre-operative high CA-125 decreased to $22 \mathrm{IU} / \mathrm{ml}$ (normal range $0-35 \mathrm{IU} / \mathrm{ml}$ ) one week after surgery.

Conclusions: Unexpectedly high CA-125 over $1000 \mathrm{IU} / \mathrm{ml}$ can be seen in benign gynecologic conditions such as severe adenomyosis. The high CA-125 level is positively correlated to the uterine size in severe adenomyosis.

Key words: severe, adenomyosis, high, CA-125, report.

\section{Introduction}

Abnormal uterine bleeding (AUB) accounts for $33 \%$ of outpatient referrals and $69 \%$ of referrals in the peri-menopausal and postmenopausal ages [1, 2]. AUB is a common cause of iron deficiency anemia (IDA), especially in the reproductive age group [3]. Polyps, adenomyosis, leiomyoma, malignancy and hyperplasia are the structural causes of AUB [3]. Endometrial biopsy is an important step in the assessment of AUB to rule out endometrial carcinoma, so that medical treatment can be offered, and unnecessary radical surgery can be avoided [1, 2].

Hysterectomy is the definitive, widely accepted treatment for fibroids, adenomyosis not responding to medical treatment and/or associated with endometrial hyperplasia [3, 4].
This case report represents a rare case of a perimenopausal woman who presented with a pelvi-abdominal enlarged uterus due to severe adenomyosis and high CA-125 to highlight that unexpectedly high CA-125 over $1000 \mathrm{lU} / \mathrm{ml}$ can be seen in benign gynecologic conditions other than malignancy.

\section{Case report}

A 46-year-old woman, with 4 previous normal vaginal deliveries, diabetic on oral hypoglycemic medications, presented to the emergency department of Ahmadi Hospital, Kuwait Oil Company (KOC), with abdominal pain and AUB over the last year.

She had received cyclic progesterone (cyclic medroxyprogesterone acetate $10 \mathrm{mg}$ daily for 10 days 
each month) with tranexamic acid (anti-fibrinolysis) 1-1.5 g 3 times/day during the bleeding episodes for the last 3 months without any response [5].

She had a tender pelvi-abdominal mass reaching two fingers breadth above the umbilicus (28 weeks' gestation). She was admitted to the hospital with the provisional diagnosis of complicated fibroid uterus for further laboratory and radiological evaluations [6].

Therapeutic curettage was performed because of the current bleeding attack, which was heavy and associated with a hemoglobin drop from 11 to $8.8 \mathrm{~g} / \mathrm{dl}[1,2]$.

The histological examination of the endometrial tissue taken during the therapeutic curettage showed cystic glandular hyperplasia without atypia.

Intravenous iron was given for correction of the patient's IDA according to the hospital protocol $[7,8]$.

The departmental trans-abdominal ultrasound of the pelvi-abdominal mass showed asymmetrical myometrial thickening, predominantly its anterior wall, with myometrial hyperechoic regions surrounded by hypoechoic areas suggestive of adenomyosis for magnetic resonance imaging (MRI) confirmation [3].

The pelvi-abdominal T2-weighted MRI confirmed the diagnosis of adenomyosis and showed a normal elongated cervix with small Nabothian follicle, diffuse asymmetrical uterine adenomyosis, predominantly its anterior myometrial wall, with an ill-defined endo-myometrial junction and both ovaries normal (Fig. 1).

Total abdominal hysterectomy with bilateral salpingectomy and bilateral ovarian conservation was performed after departmental approval and the patient's consent.

The routine pre-operative investigations including Pap smear were completely normal except the cancer

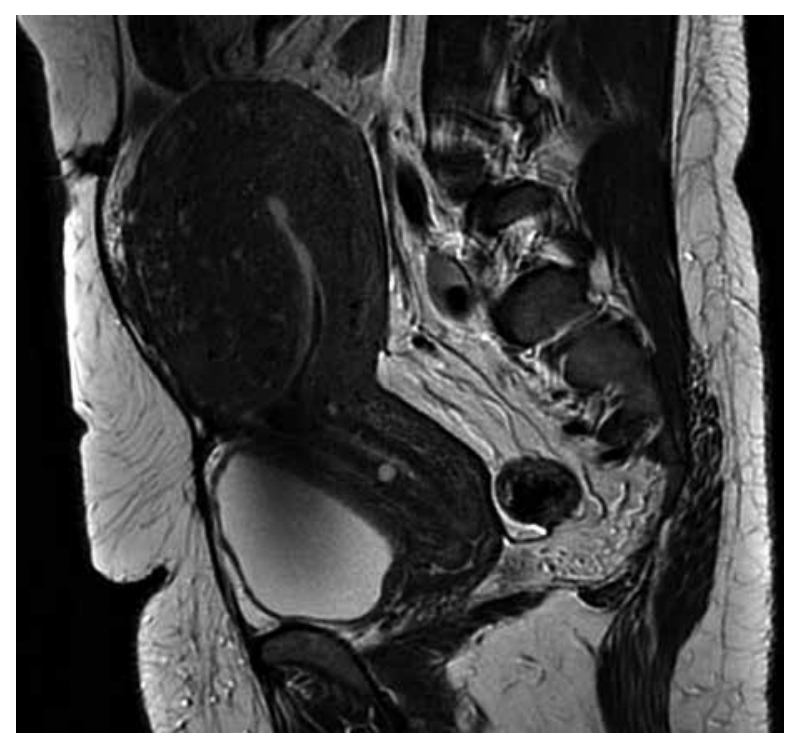

Fig. 1. Elongated cervix with small Nabothian follicle and diffuse asymmetrical uterine adenomyosis (predominantly its anterior myometrial wall) antigen (CA-125), which was unexpectedly high (1658 and $1046 \mathrm{IU} / \mathrm{ml}$ repeated twice two days apart) (Fig. 2).

The histological examination of the surgically removed uterus showed pinpoint/small cystic areas of hemorrhage seen within the myometrium with the presence of endometrial glands and stroma within the myometrium away from the endo-myometrial junction, confirming the diagnosis of adenomyosis uterus [3], and the preoperative high CA-125 decreased to $22 \mathrm{IU} / \mathrm{ml}$ (normal range 0-35 $\mathrm{IU} / \mathrm{ml}$ ) one week after surgery.

\section{Discussion}

Adenomyosis is a common pathology detected in hysterectomy specimens, characterized by the presence of endometrial glands and stroma within the myometrium [3]. Patients with adenomyosis uteri typically present with AUB at pre- or perimenopausal age.

Diagnosis of adenomyosis on clinical findings alone is usually difficult. Ultrasound is the primary imaging tool used in the evaluation of myometrial lesions [3].

The ultrasound criteria used for the diagnosis of adenomyosis are asymmetrical myometrial thickening, heterogenous area within the myometrium, hyperechoic regions surrounded by hypoechoic areas and unaltered uterine contour [3]. Myometrial cyst has been cited as the most sensitive and specific feature for the diagnosis of adenomyosis [9].

The sensitivity and specificity of transabdominal ultrasound in diagnosing adenomyosis are $32-63 \%$ and 95-97\%, respectively, while MRI has $78-88 \%$ sensitivity and $67-93 \%$ specificity in diagnosing adenomyosis [3]. MRI findings of adenomyosis include diffuse uterine wall enlargement with hyperintense foci, cystically dilated glands and sites of microhemorrhages [10].

A retrospective study was conducted by Rizvi et al. on hysterectomy specimens of patients with $A \cup B$, and they concluded that adenomyosis was the most common cause of AUB in perimenopausal age (46.34\% [38/82]) [3].

The studied woman was surgically managed by total hysterectomy and bilateral salpingectomy with bilateral ovarian conservation (because of her age of 46 years and both normal ovaries detected by pre-operative MRI and intra-operative examination) after correction of her anemia using intravenous iron, departmental approval and the patient's consent. The decision of hys-

\begin{tabular}{c|c|c|c|c}
\hline Date & Time & Result & Units & Reference \\
\hline $20 / 2 / 20$ & $13: 05$ & $1046 * \mathrm{H} Q$ & $\mathrm{U} / \mathrm{mL}$ & $0-35$ \\
\hline $18 / 2 / 20$ & $10: 30$ & $1658 * \mathrm{H} Q$ & $\mathrm{U} / \mathrm{mL}$ & $0-35$
\end{tabular}

Fig. 2. Pre-operative CA-125 was unexpectedly high (1658 and $1046 \mathrm{IU} / \mathrm{ml}$ repeated twice two days apart) 
terectomy was based on failed cyclic progesterone with tranexamic acid over the last 3 months to control the studied woman's bleeding attacks [5] and presence of cystic glandular hyperplasia detected after histological examination of the endometrial biopsy taken during the therapeutic curettage.

The studied patient had unexpectedly high preoperative CA-125 (1658 and $1046 \mathrm{IU} / \mathrm{ml}$ repeated twice two days apart) and the pre-operative high CA-125 decreased to $22 \mathrm{lU} / \mathrm{ml}$ (normal range $0-35 \mathrm{IU} / \mathrm{ml}$ ) one week after surgery.

Cancer antigen 125 (CA-125) is a high-molecular weight glycoprotein, traditionally associated with ovarian cancers, and it can be elevated in other benign conditions [11]. Kojima et al. reported elevated CA-125 $(256 \mathrm{IU} / \mathrm{l})$ in a case of adenomyosis uterus with mosaic Turner syndrome [10]. Ghaemmaghami et al. reported 3 cases scheduled for laparotomy as ovarian cancer and leiomyosarcoma due to high levels of CA-125 (> 1,000 lU/ml), and the histologic results after laparotomy showed uterine myoma in two patients and endometrioma in the third patient [12]. Ghaemmaghami et al. concluded that high levels of CA-125 (> 1,000 IU/ml) can be seen in benign gynecological condition other than malignancy [12].

Zhou et al. found that women with adenomyosis had a higher CA-125 positive rate than normal controls or those with leiomyoma and the CA-125 levels were positively correlated with uterine size [13]. In addition, Zhou et al. found that the mean CA-125 level decreased significantly one week after surgery [13].

Fifty women having uterine enlargement of $>12$ weeks' size due to severe adenomyosis and raised CA-125 were studied by Sheth and Ray, and they concluded that the greater the enlargement of the uterus over $240 \mathrm{~cm}^{3}$ volume or $>12$ weeks' uterine size due to severe adenomyosis, the greater was the rise of CA-125 levels [14].

This case report presents a rare case of a perimenopausal woman who presented with a pelvi-abdominal enlarged uterus due to severe adenomyosis and high CA-125 to highlight that unexpectedly high CA-125 over $1000 \mathrm{IU} / \mathrm{ml}$ can be seen in benign gynecologic conditions other than malignancy.

\section{Conclusions}

Unexpectedly high CA-125 over $1000 \mathrm{IU} / \mathrm{ml}$ can be seen in benign gynecologic conditions such as severe adenomyosis. The high CA-125 level is positively correlated with the uterine size in severe adenomyosis.

\section{Acknowledgments}

The authors are grateful to the studied woman, who agreed to publication of her case as a case report.

\section{Disclosure}

The authors report no conflict of interest.

\section{References}

1. Abdelazim IA, Aboelezz A, Abulkareem AF. Pipelle endometrial sampling versus conventional dilatation \& curettage in patients with abnormal uterine bleeding. J Tur Ger Gynecol Assoc 2013; 14: 40-49.

2. Abdelazim IA, Abdelrazak KM, Elbiaa AA, et al. Accuracy of endometrial sampling compared to conventional dilatation and curettage in women with abnormal uterine bleeding. Arch Gynecol Obstet 2014; 291: 1121-1126.

3. Rizvi G, Pandey H, Pant H, et al. Histopathological correlation of adenomyosis and leiomyoma in hysterectomy specimens as the cause of abnormal uterine bleeding in women in different age groups in the $\mathrm{Ku}$ maon region: A retroprospective study. J Midlife Health 2013; 4: 27-30.

4. Munro MG, Critchley HO, Broder MS, et al. FIGO classification system (PALM-COEIN) for causes of abnormal uterine bleeding in nongravid women of reproductive age. Int J Gynaecol Obstet 2011; 113: 3-13.

5. Abdelazim I, AbuFaza M, Svetlana S, Zhurabekova G. Complete amenorrhea after treatment of resistant menorrhagia using NovaSure ablation. J Obstet Gynecol Investig 2018; 1: 25-28.

6. Abdelazim IA, Abu-Faza M, Zhurabekova G, et al. Intra-leiomyoma hemorrhage in postmenopausal woman presented with acute abdominal pain. J Family Med Prim Care 2018; 7: 1129-1132.

7. Abdelazim IA, Abu-Faza M, Shikanova S, et al. Heme-bound iron in treatment of pregnancy-associated iron deficiency anemia. J Family Med Prim Care 2018; 7: 1434-1438.

8. Abdelazim IA, Nusair B, Svetlana S, Zhurabekova G. Treatment of iron deficiency and iron deficiency anemia with intravenous ferric carboxymaltose in pregnancy. Arch Gynecol Obstet 2018; 298: 1231-1232.

9. Andreotti RF, Fleischer AC. The sonographic diagnosis of adenomyosis. Ultrasound Q 2005; 21: 167-170.

10. Kojima R, Nakagawa K, Ohgi S, et al. Case report. Adenomyosis in a patient with mosaic Turner syndrome: case report. Arch Med Sci 2008; 4: 85-87.

11. Michaud Maturana M, Panayotidis I, Psarelis S, et al. Elevated CA-125 in IgG4 mesenteritis: a red herring or a disease biomarker? Case report and literature review. Rheumatol Int 2019; 39: 1285-1289.

12. Ghaemmaghami F, Karimi Zarchi M, Hamedi B. High levels of CA125 (over $1,000 \mathrm{IU} / \mathrm{ml}$ ) in patients with gynecologic disease and no malignant conditions: three cases and literature review. Arch Gynecol Obstet 2007; 276: 559-561.

13. Zhou Y, Wu B, Li H. [The value of serum CA125 assays in the diagnosis of uterine adenomyosis]. Zhonghua Fu Chan Ke Za Zhi 1996; 31: 590-593. 14. Sheth SS, Ray SS. Severe adenomyosis and CA125. J Obstet Gynaecol 2014; 34: 79-81. 